\title{
Nano-scale optical and quantum optical devices based on photonic crystals
}

\author{
Jelena Vuckovic ${ }^{*}$, Tomoyuki Yoshie, Marko Loncar, Hideo Mabuchi, and Axel Scherer \\ Caltech, Pasadena, CA 91125,U.S.A. \\ * Ginzton Laboratory, Stanford University, Stanford CA 94305-4085, U.S.A.
}

\begin{abstract}
We review our theoretical and experimental work on functional optical devices based on planar photonic crystals. Beside standard photonic applications, these structures can also be used as building blocks of quantum networks.
\end{abstract}

\section{INTRODUCTION}

Photonic integrated circuits, and quantum networks in recent years, have been in focus of an enormous research activity, motivated in part by the need to improve the speed and security of communication systems. Photonic crystals (high-reflectivity mirrors with nano-scale periodic modulation of dielectric constant in up to three dimensions) have been identified as promising candidates for both revolutionizing the integration of optical components, and for building the hardware of quantum networks. Moreover, they can be employed in construction of efficient solid-state light sources. Three-dimensional photonic crystals can be used to manipulate the flow of light in any direction in space, but they are generally very difficult to fabricate. On the other hand, when twodimensional photonic crystals are combined with highrefractive-index contrast slabs for confinement in the third dimension, as illustrated in Fig. 1., most of the functionality of three-dimensional structures can be achieved, while keeping the fabrication procedure simple. By introducing point or line defects into these planar photonic crystals, light can be localized into extremely small volumes (even smaller than a cubic optical wavelength in material), and a variety of optical devices can be constructed and integrated on a single chip.

This article reviews our work on high-quality optical nanocavities in planar photonic crystals [1-2]. Our research on photonic crystal waveguides and waveguide bends [3], and light emitting diodes based on metallic photonic crystals [4] will not be described in this paper, but will be briefly presented in the talk.

\section{HIGH QUALITY OPTICAL NANOCAVITIES IN PLANAR PHOTONIC CRYSTALS}

One of the most important properties of photonic crystals is the ability to localize light into extremely small mode volumes - even smaller than a cubic optical wavelength of light.

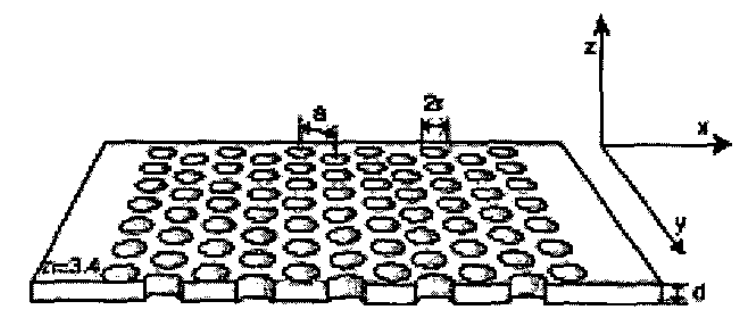

Fig. 1. Planar photonic crystal constructed by perforating an optically thin slab with a two-dimensional photonic crystal array of air holes.

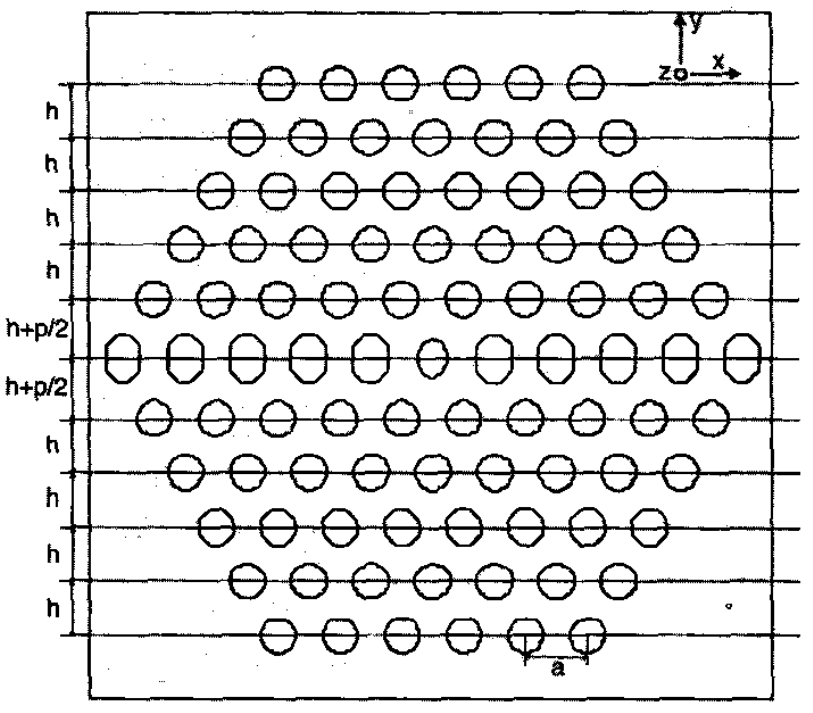

Fig. 2. Photonic crystal nanocavity with a single defect and fractional edge dislocations. Single defect is formed by reducing the radius of the central hole from $0.275 a$ to $0.2 a$ ( $a$ is the lattice periodicity). Fractional edge dislocations are produced by inserting extra material with thickness $p$ along the central row of holes. The holes in the central row become ellipsoidal, with the minor axis equal to $2 r$, and the major axis equal to $2 r+p$. Hole-to-hole distances are preserved under this transformation, as illustrated in this Figure. 


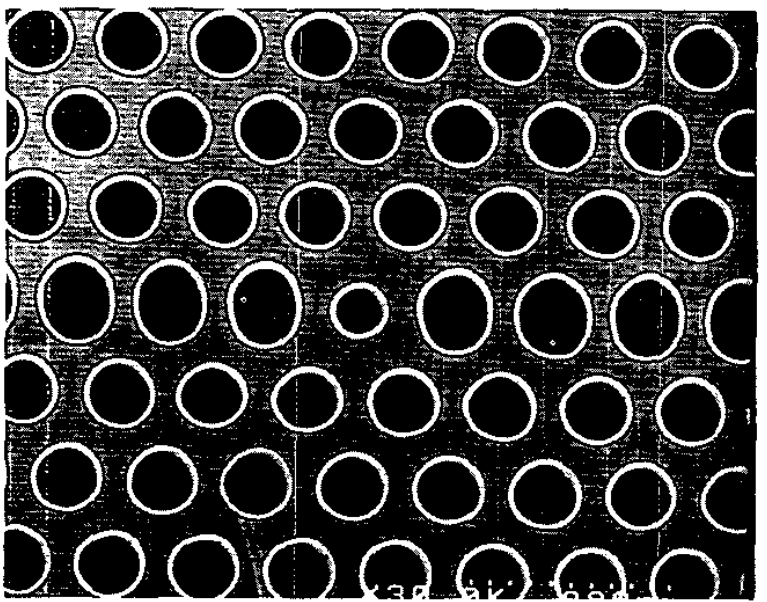

Fig. 3. Scanning electron microscope (SEM) micrograph of the planar photonic crystal nanocavity with a single defect and fractional edge dislocations. The structure is fabricated in silicon, for the operation at the target wavelength of $1.5 \mu \mathrm{m}$. The scale bar corresponds to $999 \mathrm{~nm}$.

Localization of light into such small volumes opens the possibility to construct high-density photonic integrated circuits, and to experimentally study cavity quantum electrodynamics (QED) phenomena both in the low- and high-Q regimes. These phenomena can be employed in construction of high-efficiency light-emitting diodes, lowthreshold lasers, single-photon sources, and even hardware of quantum networks and quantum computers [5]. Unfortunately, the simplest and most obvious cavity designs in planar photonic crystals suffer from poor quality factors, usually under 1000 , limited by significant losses out of the photonic crystal membrane plane, and due to the imperfect confinement by total internal reflection. Recently, we proposed novel photonic crystal cavity designs, with Q-factors larger than 10000 , together with mode volumes of the order of one half of the cubic optical wavelength in the high-refractive-index material [1]. Our cavities are based on a single defect with fractional edge dislocations. Single defect is generated by reducing the radius of a single photonic crystal air hole, or by increasing its refractive index. Fractional edge dislocations are generated by inserting a fraction of a photonic crystal lattice plane along the central row of holes, as illustrated in Fig. 2. The fabrication of these structures is as simple as a construction of any planar photonic crystal structure, and the sizes and positions of air holes are easily controlled by electron-beam lithography. The SEM micrograph of a fabricated nanocavity is shown in Fig. 3.

By tuning the elongation parameter $\mathrm{p}$, both the $\mathrm{Q}$ factor and frequency of the $\mathrm{x}$-dipole mode can be tuned, as illustrated in Fig. 4. The electric field pattern of the $\mathrm{x}$-dipole mode is shown in the inset of Fig. 4a. In this particular case, the Q-factor can be increased to around 20000 by tuning p. It is important to note that the mode volume remains roughly constant in this process, and equal to $1 / 2(\lambda / n)^{3}$, where $n$ is the refractive index of the material used to make photonic crystal $(n=3.4$ in this case).
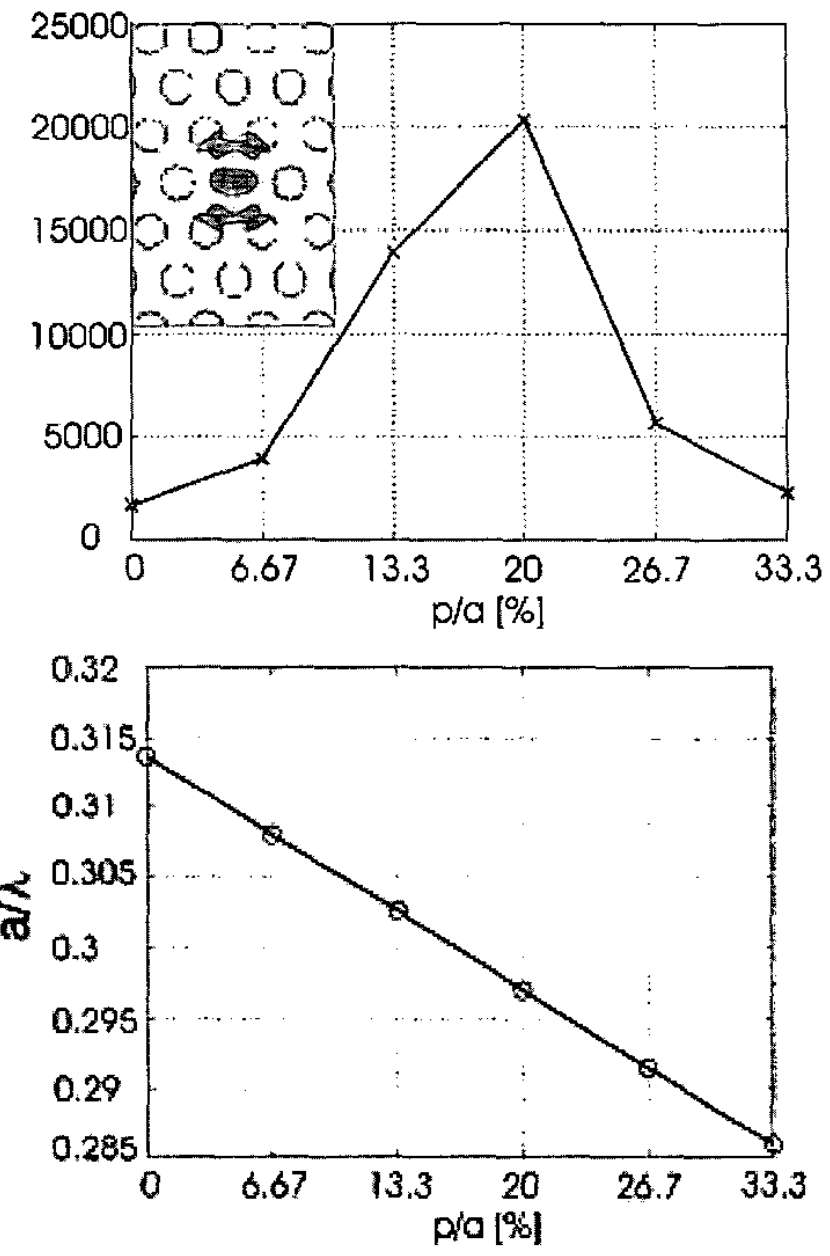

Fig. 4. Q-factor ((a), top), and normalized frequency a/ $\lambda$ ((b), bottom), as a function of the elongation parameter $p$, for the $x$ dipole mode in the structure where a single defect is produced by changing the refractive index of a single hole from 1 to 2.4 . The remaining photonic crystal parameters are $r / a=0.3, d / a=0.6$, and the refractive index of material is $n=3.4$.

We have also recently demonstrated experimentally the quality factors as high as 2800 for this type of optical nanocavity, where the theory predicted Q-factor 
of 4000 [2]. Photoluminescence from InAs quantum dots was used to probe the spectra of cavities, and our experimental results are shown in Fig. 5. Theoretically predicted behaviors of $\mathrm{Q}$ and frequency of the $\mathrm{X}$-dipole mode as a function of the elongation parameter $p$ are observed. This experimental result represents a record ratio of the Q-factor to mode volume in photonic crystals.
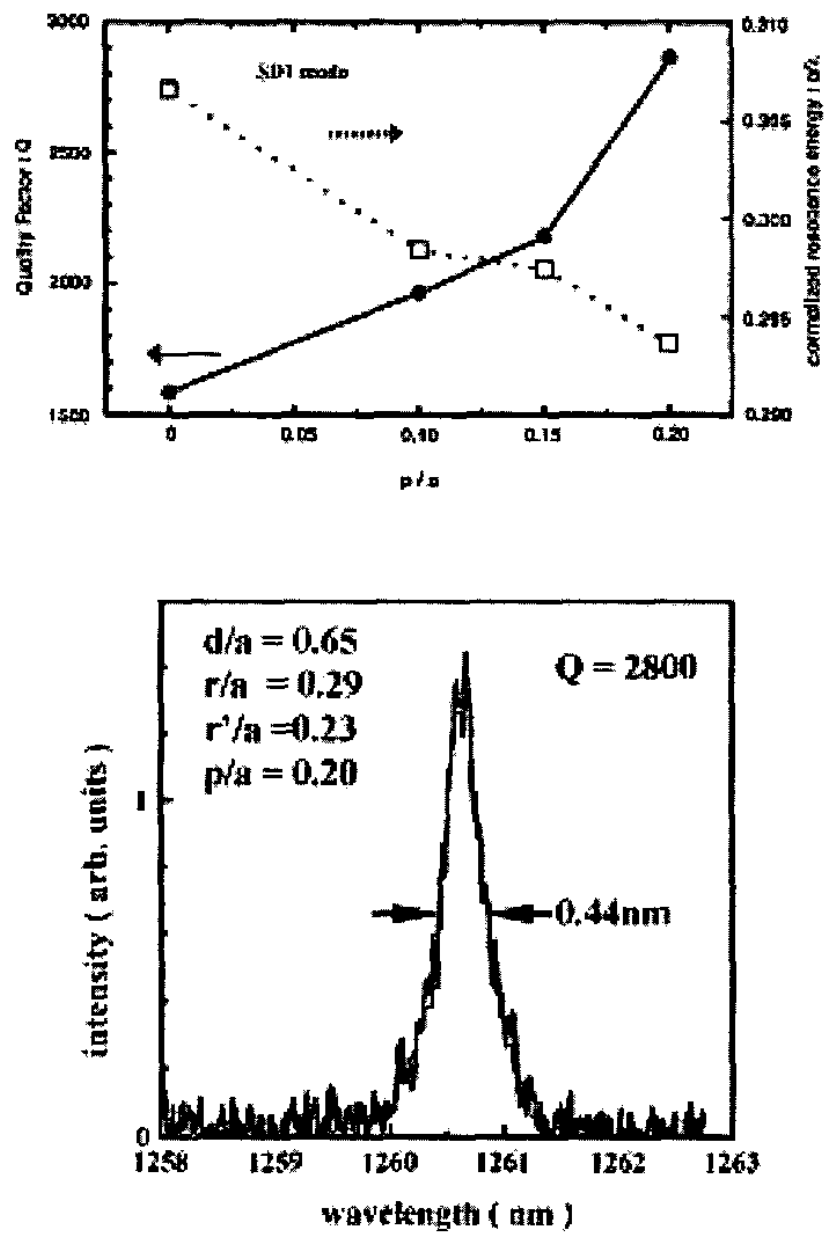

Fig. 5. ((a), top) Measured Q-factor and frequency of the $x$ dipole mode as a function of the elongation parameter $p$. The underlying photonic crystal parameters are $\mathrm{r} / \mathrm{a}=0.29, \mathrm{~d} / \mathrm{a}=0.65$, and the refractive index of material is $n=3.4$. The single defect is formed by reducing the radius of the central hole to $0.23 \mathrm{a}$. In this case, the major axis of the holes in the central row was elongated to $2 r+0.33 p$, and the minor axis remains equal to $2 r$ after the insertion of fractional edge dislocations of the order $p$. The spectrum of the structure with the peak Q-factor is shown in the bottom Figure (b).

\section{CONCLUSION}

Photonic crystals were employed in the construction of nanocavities with quality factors of 2800 , together with mode volumes equal to one half of the cubic optical wavelegth in the high-refractive index material [1-2]. In addition, waveguides with sharp bends in planar photonic crystals [3], and high-efficiency light-emitting devices based on metallic photonic crystals [4] were also demonstrated. Although the performance of these optical components can be improved further, even in their present state, they are comparable, and in some cases even better than the standard photonic components. For example, the ratio of the Q-factor to mode volume of planar photonic crystal nanocavities exceeds those achievable by standard optical microcavities, which makes them promising candidates for studying the cavity quantum electrodynamics, and for practical implementations of quantum networks and quantum computers. Moreover, planar photonic crystal components can be easily integrated in high-density photonic integrated circuits, and used in variety of applications, ranging from optical communications to quantum networking.

\section{ACKNOWLEDGEMENT}

The authors wish to acknowledge the support of Caltech MURI Center for Quantum Networks.

\section{REFERENCES}

[1] J. Vuckovic, M. Loncar, H. Mabuchi, and A. Scherer, "Design of photonic crystal microcavities for cavity QED," Physical Review E, vol. 55, article no. 016608 , January 2002.

[2] T. Yoshie, J. Vuckovic, A. Scherer, H. Chen, and D. Deppe, "High quality two-dimensional photonic crystal slab cavities," Applied Physics Letters, vol. 79, pp 42894291, December 2001.

[3] M. Loncar, D. Nedeljkovic, T. Doll, J. Vuckovic, A. Scherer, and T. P. Pearsall, "Waveguiding in planar photonic crystals," Applied Physics Letters, vol. 79, no. 13, pp. 1937-1939, September 2000.

[4] J. Vuckovic, M. Loncar, and A. Scherer, "Surface plasmon enhanced light emitting diode," IEEE Journal of Quantum Electronics, vol. 36, no. 10, pp. 1131-1144, October 2000.

[5] H. Mabuchi, M. Armen, B. Lev, M. Loncar, J. Vuckovic, H.J. Kimble, J. Preskill, M. Roukes, and A. Scherer, "Quantum networks based on cavity QED", Quantum Information and Computation, vol. 1 , special issue on Implementation of Quantum Computation, pp. 7-12, 2001 